\title{
Target Track Detection Method Based on RHT Idea in Active Sonar Image Process
}

\author{
NIE Liang Chun ${ }^{1, a}$, DENG Hong Chao ${ }^{1}$, and ZHAO Hai Xu ${ }^{1}$ \\ ${ }^{1}$ Shanghai Marine Electronic Equipment Research Institute, Shanghai, 201108, China \\ ablackman1977@163.com
}

Keywords: RHT, Image process, Track detection, similarity analysis.

\begin{abstract}
This paper proposes a target track detection method based on RHT idea in active sonar image process. The method takes advantage of speed limitation of underwater maneuvering target which means the target track distributed in local space, by means of statistical similarity analysis applied to image pixels lying on a line like curve, to make sure of pixels belonging to a line and then to accumulate in parameter space by marking the same line, with the threshold detection, it is efficiently to draw target tracks and maintain tracks' survival. This method can resolve the data gaps existed in different sonar echo ping images, and it needs not randomly sample pixels in whole image like RHT, and its realization doesn't purely rely on pixels' geometrical distribution property, i.e., more emphasis has been put on statistical similarity computation of multi-dimensional target characteristics. With these changes to standard RHT, it lowers down linearity demand in curve detection necessary in STH/RHT, and it also reduces track detection alarming rate and improve the accordance of same target track, as well as small computation burden that is favorable for noisy image track detection of large amount of pixels. This technique is very suitable for mono-stationary sonar/radar track detection in multi-ping images.
\end{abstract}

\section{Introduction}

The Hough transform(HT) was proposed by Hough in 1962[1], which was commonly used for line detection in two-dimensional binary images, and it is also called standard Hough transform(SHT) . The basic idea of SHT is that it transforms between the image space and parameter space, in which a predefined straight line passing a point in the image space is mapped to a point in the parameter space, and the line is assumed to exist and parameterized by exhaustively enumeration. Similarly, Each pixel of the image space is transformed to a parameterized curve. Each transformed point in the parameter space is considered as a candidate line, and all the pixels belonged to a line will cross in the parameter space, then it is possible to accumulate in the corresponding cell to realize curve detection by accumulation degree or marking grade management. In multi-parameter defined curve detection operation, the SHT's one-to-multiple mapping will result in large amount of computation burden, as well as mass storage demand in multi-parameter space accumulation case.

Lei Xu and Erkki Oja introduced a basic idea that combined a equal possibility random mechanism and a converging mapping mechanism into the conventional HT methods, adopting multiple-to-one mapping idea and error threshold to resolve curve parameters estimation, by means of marking or grading mechanism for same curve candidate pixels to finish curves detection. That process formed a new algorithm called the randomized Hough transform, RHT[2], for detecting curves from a binary image, Such binary images are usually obtained from grey-level images by conventional edge-detection techniques(e.g., the Sobel operator or Canny operator)。When a certain curve parameters gains enough marks or grades, it is assumed a candidate curve.

RHT method avoids heavy computation burden introduced by one-to-multiple mapping in traditional Hough transform, and it may take advantage of dynamic programming. The accumulation process in RHT is focused on parameter cell derived from multiple-to-one mapping from image space to parameter space that reduces storage demand. RHT also has such advantages as infinite parameter space, arbitrary high parameter precession, as well as low sophistication in time 
and space dimension.

SHT and RHT are all commonly used for line or curve detection in single binary image, the mark or grade accumulation is performed under given parameter dimensions, and it also uses whole image pixels sampling to draw curve spatial or geometrical distribution. In a word, SHT and RHT are suitable for single two dimension image process when there are curves in a single image.

Mono-stationary active sonar and radar image usually has only one point echo in a single ping transmission, so it is impossible to directly perform target trajectory detection by SHT or RHT; But for multi-stationary sonar or multi-resident radar image, when the multiple sensor data have been fused, there would be more than one echo points from different sensors in a single image, then it is possible to draw target trajectory in $\mathrm{x}$ axis/y axis through multi-parameter modulation by SHT or RHT。

This paper mainly consider how to draw low speed maneuver target track or trajectory in mono-stationary active sonar image. In one single ping sonar image, there is no consistent moving target track, but once multiple ping sonar images is included, SHT/RHT technique can be used to draw target tracks which are formed from maneuver target echoes in multiple ping images. When it comes to the consideration of computation efficiency and convenience, one technique based on RHT concept is proposed by this paper's author to effectively detect such low speed underwater maneuver target track or trajectory.

\section{The maneuver target echo characteristics in active sonar image}

The active sonar image is from beamformed time sequence, in which there are much clutter/interference/noise echoes. The main source of clutter is from static bottom, surface, submerged mountain stretch, and the true maneuver target echo is often flooded or buried by high clutter background. If the clutter background signal is not removed from the original sonar image, it will induce high alarming rate in target echo detection process. So at first it is necessary and important to perform clutter compression that will recognize clutter and then take it off from the considered image, only the moving target echo and few noise left.

The target echo exists in sequent multiple ping sonar image, for underwater target speed limitation from liquid sticky resistance, the targets like frogman(with or without delivery vehicle),UUV,AUV have a maximum speed less than 10 knot and it is hard to carry out underwater maneuver in short time, so the target track will distribute in a near distance field in sequent multiple ping image dimension(azimuth/range/time dimension or $\mathrm{x}$ axis/y axis/time axis), and the target echo trajectory is close to line shape. The trajectory shape and distribution is also affected by relative range to receiver sensor、 target speed transmission period from ping to ping and the stability of receiver platform. For the specialities noted above of underwater target echo trajectory, it can't directly apply RHT technique to sample the whole image pixels to realize line detection and mark accumulation, and some necessary changes should be included to basic RHT to tackle this problem.

\section{Technique for track detection based on RHT}

The RHT method is often used for line/circle/parabola detection in single image. When RHT used in binary image characteristics analysis, firstly it is necessary to get the binary edge image(by threshold detection or some standard edge detection technique such as sobel operator/canny operator). The core idea behind RHT is to estimate parameter through observation or measurement data, as well as mark/grade accumulation in the same parameter cell, with the threshold detection to realize curve detection. To finish this task, RHT needs to randomly sample the whole image and mainly take advantage of spatial or geometrical distribution of pixels, which will induce large amount of false curve declaration in highly noisy image, along with great inefficiency in pixel sampling and mark accumulation. As one know that the true target echoes are often confused or mixed up in such noisy images, and the geometrical distribution difference between pixels are not as believable as in few noisy image process. 
In the field of underwater low speed target track detection in active monostationary sonar, the basic concept of RHT should be accepted, and one line like curve can be expressed in two parameters, and the parameter estimation can be performed in a local geometrical field decided by target speed and its location relative to receiver sensor as noted above. In order to overcome the shortcomings of pure geometrical association in RHT, more dimensional parameters of target echo should be fused and combined into a whole statistical mathematic expression to check the similarity between pixels from sequent ping echo images. The parameter vector should include such information as target azimuth, range, strength, radial scale size, numeric echo structure, etc. Through data association based on similarity computation of multidimensional parameter vector, it is much more truly to declare there is a line like curve existed in a series of given multiple ping images when the similarity computation results exceed a fixed threshold .

A line like curve distribution can be expressed by two parameter, as noted as $y=k x+b$. When the line crosses one pixel location $(\mathrm{x} 0, \mathrm{y} 0)$ in a two dimensional image, given a slope parameter $\mathrm{k}$, then $\mathrm{b}$ would be settled down. For two time sequent ping images numbered as $k$ (The newest ping)and $\mathrm{k}-1$ (the history ping, and $\mathrm{k}=0$ means the time start ), firstly two pixels from ping $\mathrm{k}$ and $\mathrm{k}-1$ should be picked up to express a possible line and resolve the parameter $(\mathrm{k}, \mathrm{b})$, assuming that the target track is near line shape(which is true in most real conditions)and the linearity would last as long as $\mathrm{N}$ ping in time domain, then the task of line detection in sequent ping image would be simplified as to seek if there is a line like curve in ping $\mathrm{k}, \mathrm{k}-1, \mathrm{k}-2, \ldots, \mathrm{k}-\mathrm{N}+1$ image. The line search process would be described as spatial or geometrical same target pixels searching in near space field among sequent images, and the techniques such as statistical similarity analysis , threshold detection , M/N rule ( $\mathrm{M}$ true value in $\mathrm{N}$ instances)would be included. To include the statistical similarity analysis can loosen the linearity demand when there is displacement away from the perfect line predefined, and one pixel should be regarded as a point to a line once it conforms to a certain statistical similarity threshold rule, not just by geometrical association as in RHT, which is very important for real data analysis, and it can resolve the image fluctuation bias.

Before the target echo line detection starts up, clutter compression or noise reduction should be performed to eliminate major static underwater target echoes and random noise background, then the left pixels should be only moving target echoes pixels as well as a few noise pixels, following that an accumulation period of $\mathrm{N}$ ping cleaned image should be performed. Clutter compression or noise reduction is finished as following: Through continuous dynamic filtering of sonar image, the slow varying background image would be estimated, with dynamic detection thresholds for different clutter types such as clump clutter, single point clutter or pure noise, a cleaned image would be derived by subtracting the background image from current considered image.

The whole target echo track curve or trajectory detection process would be illustrated in following steps.

Step one:

To check if there is similar echo pixel between current ping $\mathrm{k}$ and history ping $\mathrm{k}-1$ in near geometrical field, the first pixel from ping $\mathrm{k}$ should be considered as possible echo, and the second pixel should be searched from ping k-1. Two pixels can be used to express a line as parameter pair(k,b). All local pixels in ping k-1 would be kept as candidate points belonging to true lines associated the considered target echo pixel in current ping $\mathrm{k}$, and the search domain size(in range and azimuth dimension ) would be determined by assumed maximum target speed and its location relative to receiver sensor. Assume in step one there would be $L$ line candidates which cross a same point pixel in ping $\mathrm{k}$, and one don't know which one is really true.

Step two:

Base on step 1 results of $L$ line candidates, expressed as (kn,bn)parameter vector, $n=1,2, \ldots, L$; Now the work is to make sure whether the $\mathrm{L}$ line have measured pixel candidates among sequent ping $\mathrm{k}-2, \mathrm{k}-3, \ldots, \mathrm{k}-\mathrm{N}+1$.If there are candidate pixels assumed to belong to a line, then a corresponding mark or grade counter will add 1 automatically to the line cell. The decision of a pixel belonging to a line is heavily relying on target echo's multidimensional parameters similarity computation and later 
threshold detection, in another word, a mathematic expression would be constructed according to multi-parameter $\chi 2$ distribution function, which needs parameter estimation standard error value and the expected parameter value. A similarity assertion should be made when the $\chi 2$ based statistical expression value is less than a given threshold which is determined by confidence degree, which means all the pixels near the line would be assumed to belong to a same target if they pass the statistical test. For fulfilling this test, a line prediction and filter operator would be included, such as a simple alpha-beta filter is suitable to be adopted in this application.

Step three:

When the line similarity computation is finished between the current ping $\mathrm{k}$ image and history ping ping $\mathrm{k}-1, \mathrm{k}-2, \ldots, \mathrm{k}-\mathrm{N}+1$ images for a given pixel in ping $\mathrm{k}$, as well as mark or grade accumulator, a $\mathrm{M} / \mathrm{N}$ rule would be taken to find whether a possible true line is existed. There may be more than one line kept after the threshold detection for the pixel of ping $\mathrm{k}$.

Step four:

For the pixel in current ping $\mathrm{k}$ image, the line with highest mark or most measured point pixels would be finally assumed as a true line with high confidence, and the line passing through this measurement point pixel has been successfully extracted as a true echo pixel. As a result, this point pixel would be kept as a line's new measurement value, and the noise or clutter would be kicked out.

For all pixels in current ping k image after clutter compression or noise reduction, step one to four process would be repeated to find the most possible lines in the $\mathrm{N}$ sequent history ping images.

Step five:

For all the lines extracted after step four operation, in the next step it needs to find their new measurements in next ping $\mathrm{k}+1$ 。 If there exists a line's new measurement, then the verified measurement pixels would be kept, whereas one line couldn't find its new measurement pixel, then a check process is initiated. The check process is used to make sure if the line is pseudo line or it just has a data gap on the line, for the first case, the false line would be deleted, and for the latter case, the data gap would be repaired by prediction value of line parameters.

Step six:

After step five process, the left pixels of the next ping $\mathrm{k}+1$ image which don't match any existed lines would start up step one to four operation to find new possible lines, in which ping numbered as $\mathrm{k}+1, \mathrm{k}, \mathrm{k}-1, \ldots, \mathrm{k}-\mathrm{N}+2$ image data would be included.

With the help of step one to six process, all possible true lines or line like curves would be picked up in sequent $\mathrm{N}$ ping images.

The method proposed here adopts the core idea of RHT ,i.e, the mapping connection between measurement space and parameter space, as well as mark/grade mechanism for same line. But the new method doesn't need to randomly sample the whole image to find possible lines, and there is no step like parameterization sorting in parameter space. With the inclusion of target echo's similarity analysis realized by statistical computation based on multidimensional parameters, it doesn't purely rely on pixel's geometrical distribution to assert a line like curve, and the target track line detection confidence rating has been improved, as well as low alarming rating gained. Finally the new method doesn't demand rigid linearity when applying two parameter line RHT like process, and it is suitable for line like curve detection.

Particularly, In this paper for underwater moving target echo track detection, a maximum speed of about 10 knots limitation is set, that guarantees small search geometrical field in sequent ping images, reducing data computation burden, and it can realize some Track-before-detect effect in image process.

\section{Data analysis and results}

Using the method based on RTH idea which is discussed in section 3 of this paper, one can start up the work of how to extract slow moving target's trajectory in sequent active sonar images. Firstly the background noise and clutter or interference should be reduced or compressed, only the moving target echo pixels should be reserved, as well as a few noise which is hard to deleted entirely . After 
the clutter compression or noise reduction is performed, the amount of computation can greatly be reduced, as well as the false alarm rate coming from false pixel association.

Some actual sea trial sonar image data from an active image sonar is processed by applying the method mentioned above, with the range scope of the target speed search from -10 knot to 10 knot, and the local spatial search field is decided by the distance of the target to the receiver and the sonar ping transmission period. In noise reduction period a local SNR threshold of $-6 \mathrm{~dB}$ is applied, and the target track line or trajectory in sequent 10 ping duration time is considered to obey near linear distribution.

Initial sonar image before noise reduction is shown in figure 1, it is a 10 ping sonar image combination display, called history ping display, and it has time-distance-angle information. Near spatial location $\left(52^{0}, 780 \mathrm{~m}\right)$ there is a approximate straight line trajectory formed in the vicinity of the target echo location. Large amount of interfere or clutter and noise can be seen in the original image.

Before Noise Reduction

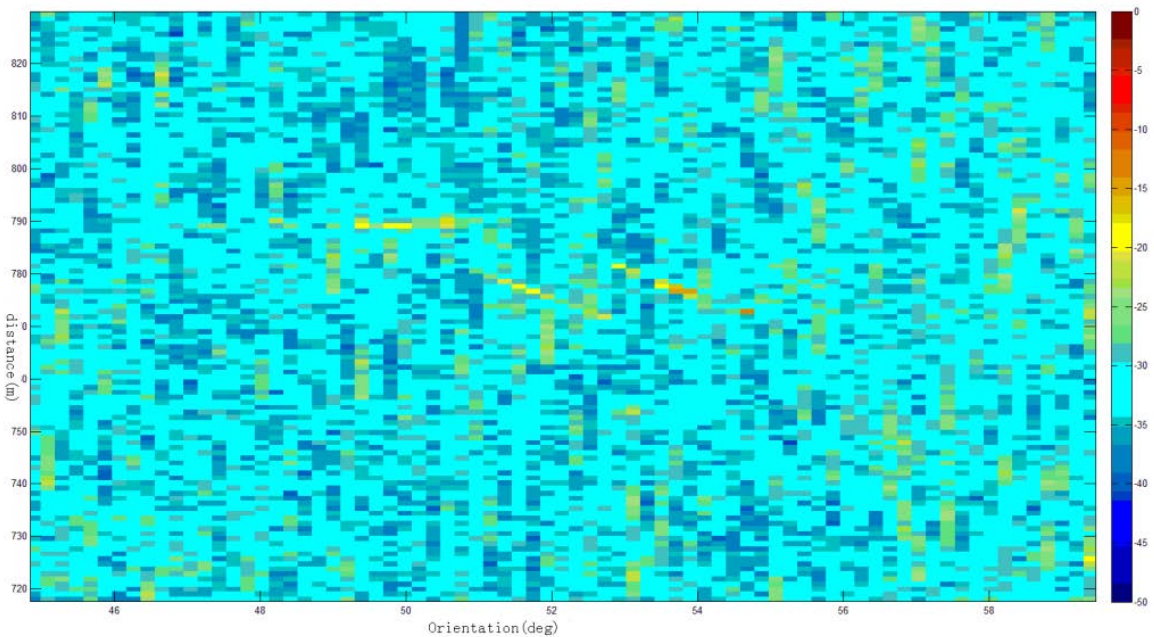

Fig. 1 image before noise reduction

The figure 2 displays the sonar image after noise reduction(the clutter or interference signal have been restrained) of figure 1.Compared with the figure 1, one can find the background noise have mostly be reduced in figure 2, and the main target echo pixels have been reserved, which formed line like target trajectories between multiple sequent ping, and there are some data gap on the lines.

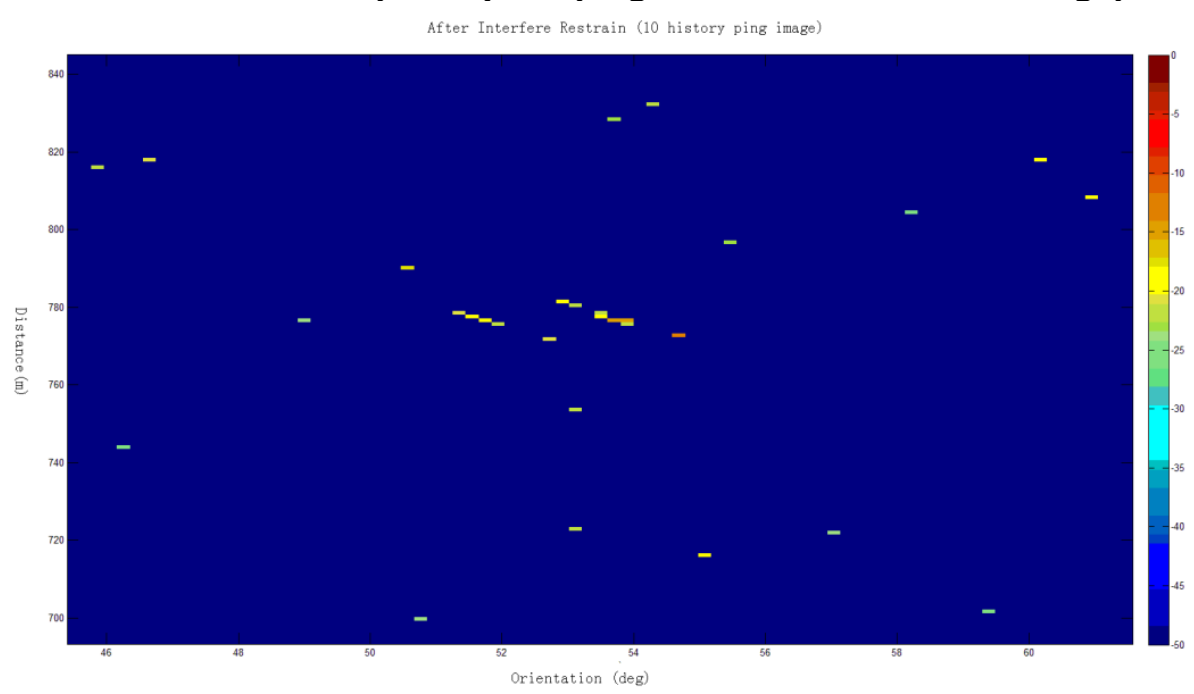

Fig.2 image after noise reduction

Applying the method proposed in this paper to figure 2 image(which is only a zoom-in of a local image to illustrate the process effect), local line detection results in sequent 10 ping image are displayed in figure 3. As one can find in figure 3 that more target line like trajectories have been drawn out than that displayed in fig.2. The reason is that the target maneuvered from near to faraway, from left to right direction(moving far away from small orientation to large orientation), and there 
were a lot of data gap around the main echo beam direction which would be from side lobe echoes. After the track line detection process, all the trajectories have been picked up, as well as data gap mended up.

The target's estimated true trajectory is shown in dash-point line in figure 4, which displays a whole maneuvering process from the slow moving target's startup to the current point pixel location. From figure 4 one can clearly see that the history echo pixels in the left part are gradually fading out, but the new echoes on the right side are growing stronger step by step. The data gap formed by observation image data missing are all filled out by line prediction values, which results a much clear target trajectory picture.

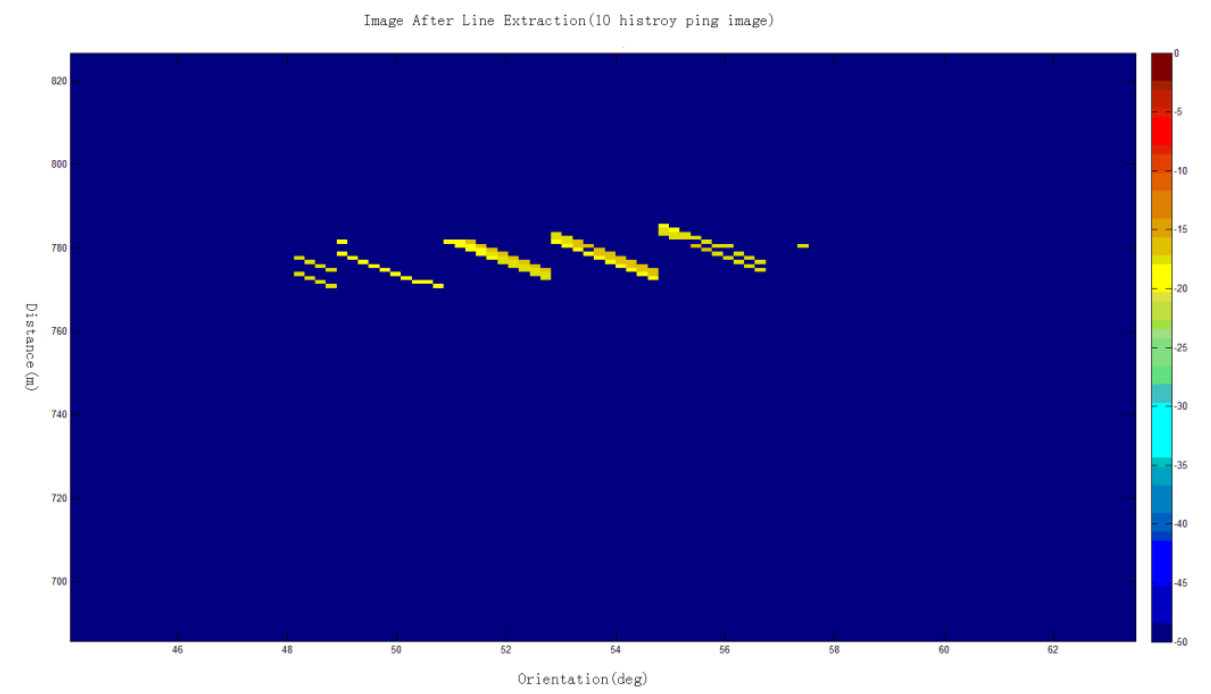

Fig.3 image after noise reduction

Image After Line Extraction(10 histroy ping image)+Trajectory

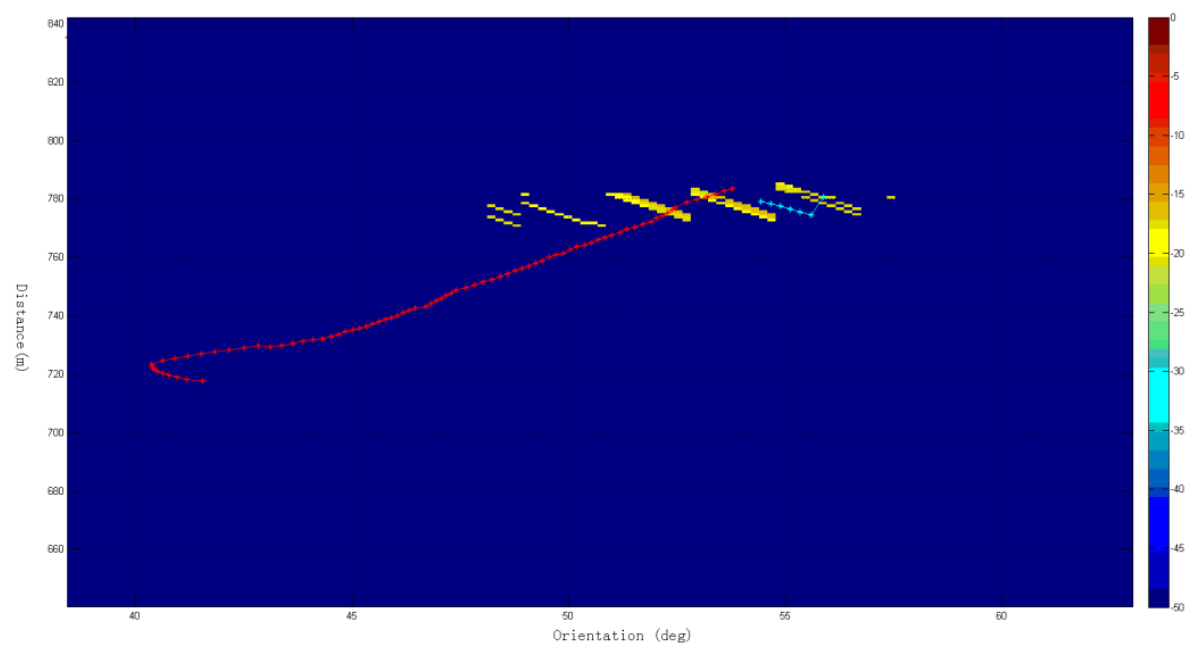

Fig.4 target’s line like trajectory detected (trajectory :dash point line, target is moving away)

\section{Summary and further work}

In this paper, a RHT based line like curve detection method has been discussed, under the assumption that in short time period the target's trajectory is approximate to line distribution ,and target moving speed is limited to a reasonable scope range. With introduction of statistical similarity analysis method combined together with threshold detection, a target echo's image pixels from sequent ping image are verified and its mark or grade is accumulated, then a local line like curve is successfully drawn out by $\mathrm{M} / \mathrm{N}$ rule, which can result in a long time survival target trajectory. Through the real sea trial data analysis and line like curve picking processing ,it is proven that the target trajectory buried in sequent multiple ping images can be efficiently extracted, at the same time, the measurement data missing or data gap of target echo between different pings can be amended up 
by line prediction and filtering. The method is very suitable for target trajectory's extraction of single sonar or radar image if multiple ping images in time sequence are available .

This method based on RTH idea does not require full-screen pixel searching. With the introduction of statistical target parameter similarity calculation and threshold detection, it can greatly reduce the probability of false alarm in target trajectory extraction and it also greatly improve the accordance of same target track line test.

The next step work will be focused on elimination of false line detection, and it needs design more effective statistical data association logic, as well as sustained and effective management of the moving target trajectory.

\section{References}

[1] P.V.C Hough, in:Method and Means for Recognizing Complex Paterns,U.S.Patent 3069654(Dec.18,1962).

[2] Xu,L.and Erkki.Oja(1993),in:Randomized Hough Transform(RHT) : Basic mechanisms, Algorithms, and Computational Complexities, Comput.Vision Graphics Image Process.:Image Understanding, 57(2),131-154. 